\title{
Sistema Web De Avaliação Da Produção Científica Brasileira (SWAP-BR)
}

\author{
Felipe O. Simões ${ }^{1}$, Jacques Dúlio Brancher ${ }^{1}$ \\ ${ }^{1}$ Departamento de Ciência da Computação - Universidade Estadual de Londrina \\ Caixa Postal 10.011 - 86.057-970 - Londrina - PR - Brasil \\ felipeoliveirasimoes@hotmail.com, jacques@uel.br
}

\begin{abstract}
Due to the importance of analyzing curricula for decision-making in educational institutions, development agencies and research groups, it becomes necessary ways to evaluate them quickly, transparent and fair. Due to this need, SWAP-BR was developed. It consists of a system capable of evaluating in a broad and automated way the Currículo Lattes of teachers and researchers. For this it uses the Pontuação Qualis (PQ) and Rep-Index as evaluation metrics. In addition SWAP-BR can be adjusted to work with several areas of knowledge, taking into account characteristics of each. Although, he is able to receive the information and parameterizations of the different areas, this paper will only present the results obtained for the area of Computer Science. It can also offer greater transparency in selective processes or evaluation of meritocratic character, which involves analysis of curricula or performance of researchers.
\end{abstract}

Resumo. Devido a importância da análise de currículos para tomadas de decisões de instituições de ensino, agências de fomento e grupos pesquisa, cada vez mais, torna-se necessário maneiras de avaliá-los de forma rápida, transparente e justa. Devido a esta necessidade, foi desenvolvido o SWAP-BR. Que consiste em um sistema capaz de avaliar de maneira ampla e automatizada o Currículo Lattes de docentes e pesquisadores. Para isto ele utiliza a Pontuação Qualis $(P Q)$ e o Rep-Index, como métricas de avaliação. Além disto o SWAP-BR pode ser ajustado para trabalhar com diversas áreas do conhecimento, levando em consideração as características de cada uma. Porém, embora ele esteja apto a receber as informações e parametrizações das diversas áreas, neste trabalho serão apresentados apenas os resultados obtidos para a área de Ciência da Computação. Com ele também é possível oferecer maior transparência em processos seletivos ou de avaliação de cunho meritocrático, que envolvam análise de currículos ou desempenho de pesquisadores.

\section{Introdução}

No âmbito acadêmico, a avalição de currículos é utilizada para selecionar e compor o corpo docente de cursos e programas, para destinar bolsas e recursos para projetos, no processo seletivo para o ingresso de alunos em programas de mestrado e doutorado, e pela Capes na hora de avaliar programas brasileiros de pós-graduação [CAPES 2013b]. Tendo em vistas a importância da avaliação de currículos, o CNPq desenvolveu a Plataforma Lattes, com o objetivo de armazanar, centralizar e divulgar os chamados Currículos Lattes.

Estes por sua vez, se tornaram um padrão nacional no registro da vida pregressa de estudantes e pesquisadores de todas as áreas do conhecimento [CNPq 2016]. Embora 
a Plataforma Lattes padronize e disponibilize os currículos, ela por si só, não é capaz de atender a todas as demandas e necessidades de pesquisadores e instituições, como por exemplo, identificação do conceito Qualis das publicações de cada indivíduo, geração de gráficos e estáticas e uma avaliação automatizada de grupo de currículos.

Desta forma surgiram ferramentas como o ScriptLattes [Mena-Chalco et al. 2009], que tenta suprir partes destas necessidades. Este por sua vez consiste em um sistema de prospecção de dados do Currículo Lattes, com o objetivo facilitar a análise e avaliação de um ou mais currículos. Porém ele não fornece meios de avaliar de maneira rápida e direta o desempenho dos pesquisadores, fazendo com que este trabalho seja realizada de maneira manual.

Tendo em vista as necessidades apresentadas, este trabalho descreve o SWAP-BR, que trata-se de um sistema, que possibilita a análise e avaliação automática do Currículo Lattes de pesquisadores da diversas áreas do conhecimento. A principal vantagem em sua utilização em relação às demais ferramentas existentes no mercado, é a implementação do Rep-Index como métrica de avaliação. Que ao contrário de outras métrica como, H-Index [Hirsch 2005], G-Index [Egghe 2006] e fator de impacto do JCR [Garfield 2010], ele tem por objetivo avaliar os diversos aspectos da vida profissional de um pesquisador, como orientações, titulação, bancas e publicações [Cervi et al. 2011].

Outra vantagem oferecida pelo SWAP-BR é a transaparência proporcionada nas avaliações dos currículos, nos processos seletivos de candidados a discentes ou docentes de programas de pós-graduação, para distribuição de bolsas de produtividade fornecidas pelo CNPq, e na avalição de programas de pós-graduação realizado pela CAPES. Isto porque além do sistema mostrar de forma detalhada a nota de cada um dos aspectos avaliados, é possível compartilhar as informações de forma a torná-las públicas.

\section{Modelos e Métricas de Avaliação}

Ferramentas que possuem como propósito avaliar ou classificar publicações, ou a reputação e desempenho de pesquisadores, como é o caso do ScriptLattes [Mena-Chalco et al. 2009], Google Acadêmico [Google 2004], Microsoft Academic [Microsoft 2012] e PageRank [Page et al. 1999], todas utilizam-se de modelos e métricas de avaliação. No SWAP-BR os modelos e métricas de avaliação utilizados foram, a Pontuação Qualis e o Rep-Index em conjunto com Rep-Model.

\subsection{Pontuação Qualis}

Tem por objetivo medir a qualidade e a quantidade da produção intelectual de um pesquisador em um determinado período de tempo. Para isto é necessário obter o conceito Qualis de cada uma das publicações pertencente a determinado pesquisador.

Porém o Qualis não avalia diretamente as publicações, e sim os veículos de divulgação nos quais estes trabalhos foram aceitos [CAPES 2013b]. Desta forma a Pontuação Qualis afere a qualidade de um trabalho baseado na qualidade do seu local de publicação. Mesmo método utilizado pela Capes em suas avaliações.

Para a área de Ciência da Computação, a Capes mantém uma lista com o Qualis tanto de periódicos, quanto de conferências. Ela entende que para a área, as conferência possuem uma relevância semelhante aos periódicos, já que muitas possuem rigorosos 
critérios de avaliação. Sendo tradicionalmente utilizados por pesquisadores da área, para a publicação de seus trabalhos [CAPES 2016a].

A Capes avalia todas os veículos de divulgação nos quais houveram publicações relacionadas a docentes de programas de pós-graduação, e submetidos dentro do sistema de coleta de dados chamado Sucupira [CAPES 2016b]. O intuito é classificá-los com algum dos seguintes conceitos Qualis: A1, A2, B1, B2, B3, B4, B5 e C [CAPES 2013b].

Além da normalização em estratos ou conceitos, a Capes também atribui a cada um deles um valor inteiro, que varia entre 0 e 100 de acordo com o grau de relevância de cada um [CAPES 2013a]. A Tabela 1, mostra a relação entre cada um dos conceitos com suas respectivas notas.

Tabela 1. Pontuação dada a cada um dos estratos do Qualis.

\begin{tabular}{|c|c|c|c|c|c|c|c|c|}
\hline Qualis & A1 & A2 & B1 & B2 & B3 & B4 & B5 & C \\
\hline Nota & 100 & 85 & 70 & 55 & 40 & 25 & 10 & 0 \\
\hline
\end{tabular}

A partir dos valores mostrados na Tabela 1, é possível obter o indíce Pontuação Qualis (PQ) para cada pesquisador. Para isso é necessário identificar o Qualis de cada uma das publicações que serão avaliados em determinado período de tempo, e então realizar o somátorio das notas referentes a seus respectivos Qualis.

\subsection{Rep-Model}

O Rep-Model foi criado de forma a mapear as diversas atividades inerentes a trajetória acadêmica de pesquisadores das diferentes áreas do conhecimento [Cervi et al. 2013a]. Para isso ele identifica e mapeia um total de 17 elementos, divididos em 5 categorias. Todos eles presentes no cotidiano da maioria dos pesquisadores e facilmente identificáveis no Currículo Lattes. A Tabela 2 mostra os elementos e categorias do Rep-Model.

Tabela 2. Rep-Model - Elementos e Categorias. (Fonte: Adaptado [Cervi 2013])
\begin{tabular}{|l|l|l|}
\hline Categoria & Elemento & Sigla \\
\hline \multirow{3}{*}{ Identificação (ID) } & Nome & NM \\
\cline { 2 - 3 } & Instituição & INST \\
\cline { 2 - 3 } & Grau de Instrução & GI \\
\hline \multirow{3}{*}{ Orientação (ORI) } & Orientação de Mestrado & OM \\
\cline { 2 - 3 } & Orientação de Doutorado & OD \\
\cline { 2 - 3 } & Orientação de Pós-doutorado & OP \\
\hline \multirow{2}{*}{ Banca (BAN) } & Participação em Banca de Mestrado & PBM \\
\cline { 2 - 3 } & Participação em Banca de Doutorado & PBD \\
\hline \multirow{3}{*}{ Comitê (COM) } & Membro de Corpo Editorial de Periódico & MCEP \\
\cline { 2 - 3 } & Revisor de Periódico & RP \\
\hline \multirow{5}{*}{ Publicação (PUB) } & Artigo em Periódico & AP \\
\cline { 2 - 3 } & Capítulo de Livro & CLIV \\
\cline { 2 - 3 } & Livro & LIV \\
\cline { 2 - 3 } & Trabalho Completo em Conferência & TCC \\
\cline { 2 - 3 } & Pontuação Qualis & HI \\
\cline { 2 - 3 } & Projeto de Pesquisa & PP \\
\cline { 2 - 3 } & Software & SOFT \\
\hline
\end{tabular}


O mapeamento destes 17 elementos, com a possibilidade de agregar novos elementos caso necessário, garante a adaptabilidade deste modelo. Já que desta forma ele é capaz de mapear aspectos da vida profissional de pesquisadores de diferentes áreas. Porém para que a avaliação de um pesquisador seja de fato coerente com sua área, é utilizado um sistema de ponderação de pesos para cada um deste elementos e categorias [Cervi et al. 2013b].

Desta forma cada elemento e categoria recebe um peso coerente com a relevância que ele possui para a área de avaliação. Sendo assim é possível atribuir pesos diferentes para o mesmo elemento. Então se para uma área, um determinado elemento se mostra bastante relevante, basta atribuir um peso maior para ele, enquanto nas demais, ele pode vir a possuí um peso significativamente menor.

\subsection{Rep-Index}

O Rep-Index consiste em um índice que quantifica a qualidade ou a reputação do trabalho de um pesquisador. Para isso ele utiliza o Rep-Model e seu sistema de pesos para saber quais elementos deverão ser considerados durantes os cálculos e a relevância que cada um deles possui para a área do conhecimento [Cervi et al. 2012].

Sendo assim o cálculo para se chegar ao valor do Rep-Index, é realizado a partir da somatória do valor de cada elemento presente no Rep-Model, multiplicado por seus respectivos pesos, juntamente com a somatória de cada categoria, também considerando seus pesos. A Equação 1 exemplifica os cálculos realizados para se alcançar o Rep-Index.

$$
R e p-\operatorname{Index}_{(R)}=\sum_{i=1}^{c}\left(\sum_{j=1}^{e_{i}} \frac{v_{j} * w_{j}}{\max \left(v_{j}\right)}\right)
$$

- R: Refere-se ao pesquisador que se quer identificar a reputação;

- c: Representa o número total de categorias;

- i: Representa o intervalo de 1 até o número total de categorias (c);

- $\mathrm{e}_{\mathrm{i}}$ : Representa o número total de elementos;

- j: Refere-se ao intervalo de 1 até o número total de elementos (ei);

- v: Representa o valor do elemento;

- $\mathrm{w}_{\mathrm{j}}$ : Refere-se ao peso do elemento;

- $\max \left(\mathrm{v}_{\mathrm{j}}\right)$ : Representa o maior valor do elemento.

Analisando a Equação 1, pode-se observar que cada elemento é multiplicado por seu peso e dividido pelo maior valor apresentado para aquele determinado elemento. Desta forma o Rep-Index gera um valor real e inferior a 100 [Cervi et al. 2013b]. Este valor reflete o desempenho do pesquisador em determinado período de tempo e de acordo com a área de avaliação.

\section{SWAP-BR}

O SWAP-BR consiste em um sistema web, com o objetivo de automatizar o processo de avaliação de Currículos Lattes. Para isso ele utiliza-se de modelos e métricas que reflitem de maneira quantitativa a qualidade e reputação do trabalho de um pesquisador, ao longo de sua carreira ou em um determinado período de tempo. 
Ele foi criado para ser um sistema modular, de forma que módulos possam ser acrescentados ou removidos, sem a necessidade de grandes alterações no sistema [Khaliluzzaman and Chowdhury 2016]. Ele consiste em um sistema com 8 (oito) módulos e 35 (trinta e cinco) funcionalidades distintas relacionadas a eles. O diagrama mostrado na Figura 1, consiste no diagrama de caso de uso de alto nível do SWAP-BR. Nele são mostrados módulos e funcionalidades presentes no sistema.

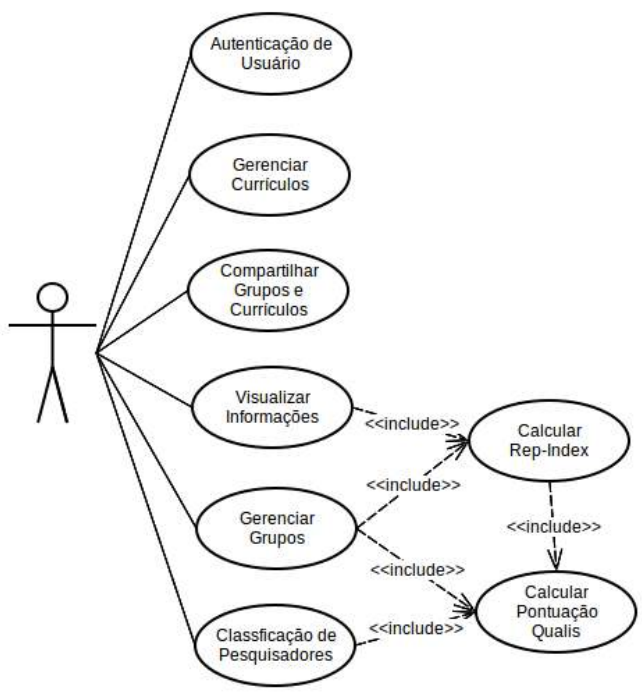

Figura 1. Diagrama de alto nível do SWAP-BR.

O diagrama da Figura 1 representa a interação do usuário com o sistema. Desta forma é mostrado apenas os módulos e funcionalidades com as quais o usuário interage diretamente. Sendo assim os demais módulos e funcionalidades que não aparecem no diagrama, são utilizados de forma indireta por outros. Todos os módulos estão descritos abaixo:

- Autenticação de Usuário: Responsável por gerenciar o cadastro e autenticação do usuário no sistema, e realizar o controle de acesso a páginas e funcionalidades.

- Gerenciar Currículos Lattes: Cabe a ele gerenciar toda a parte de inclusão e remoção do Currículo Lattes no sistema.

- Calcular Pontuação Qualis: Possui a função de identificar o conceito Qualis de cada publicação realizada pelo pesquisador, de acordo com a área de conhecimento. Após isso realiza os cálculos para se alcançar o valor da Pontuação Qualis.

- Calcular Rep-Index: Realiza os cálculos necessários para gerar o Rep-Index. Para isto são obtidos os maiores valores para cada elemento, e são calculados o valor para cada elemento e categoria que serão avaliados.

- Gerenciar Grupos: Responsável por realizar todo o gerenciamento que envolva a criação, exclusão de grupos. Adicionar e remover pesquisadores ao grupo. Além de mostrar estatísticas, gráficos e avaliações correspondente ao grupo.

- Compartilhar Currículos e Grupos: O SWAP-BR permite que currículos e grupos de um determinado usuário seja compartilhado com outros. Este módulo é responsável por gerenciar toda a parte do compartilhamento dentro do sistema.

- Classificação de Pesquisadores: A classificação ou raking de pesquisadores, permite a criação de uma lista de pesquisadores, a partir da Pontuação Qualis de cada 
um. Leva em consideração as áreas e subáreas do conhecimento e o período de tempo analisado.

- Visualizar Informações dos Currículos: Responsável por gerenciar a organização e layout de as informações que serão exibidas para o usuário. Este módulo é o encarregado de gerar a maioria das tabelas e gráficos presente no sistema.

\section{Experimentos e Resultados Obtidos}

Com o objetivo de validar o SWAP-BR, foram realizados alguns testes e experimentos nos quais pode-se constatar que de fato ele supre a necessidade para a qual ele foi desenvolvido, permitindo assim uma avaliação rápida, transparente e coerente com a área de conhecimento avaliada.

Com o objetivo de analisar o desempenho do processo de identificação do Qualis, foi realizado um teste onde verificou-se a taxa de publicações classificadas corretamente, tanto para Artigos em Periódicos (AP) quanto para Trabalhos Completos em Conferências (TCC). Para isso analisou-se o currículo de 47 pesquisadores escolhidos aleatóriamente, dentre os 469 existentes na base de dados. Estes por sua vez correspondem aos docentes de programas de pós-graduação da área de Ciência da Computação, cujo os conceitos variam entre 5 e 7.

Para Artigos em Periódicos (AP), observou-se que 1067 publicações tiveram seus respectivos Qualis classificados corretamente. Outras 52 publicações não tiveram seus Qualis identificados, devido aos periódicos nos quais foram publicados não constarem nas lista da Capes contendo os Qualis de periódicos.

$\mathrm{O}$ restante das publicações em $\mathrm{AP}$, não puderam ter seus devidos Qualis identificados, devido a erros no preenchimento do ISBN, contendo inclusive alguns ISBN em branco. O gráfico mostrado na Figura 2 mostra o resultado para a classificação dos Artigos em Periódicos (AP).

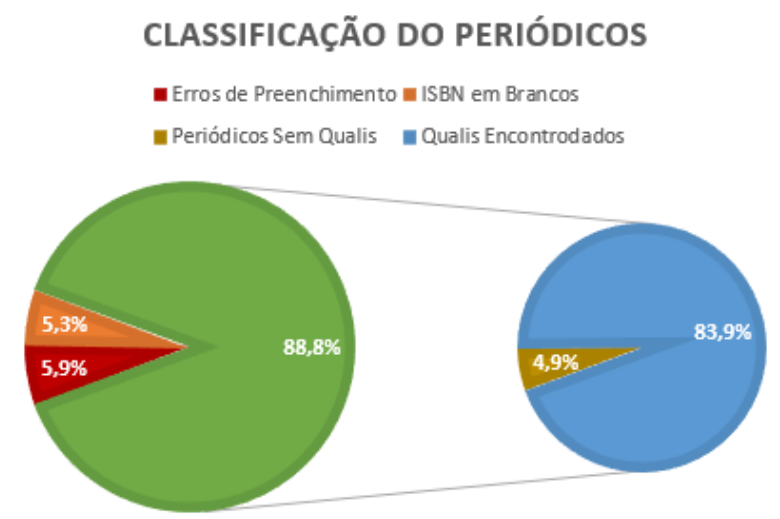

Figura 2. Classificação do Qualis para Artigos em Periódicos.

Analisando a Figura 2 pode-se afirmar que o processo de identificação dos Qualis dos Artigos em Periódicos (AP), utilizando o ISBN como método de comparação, é bastante eficiente e confiável. Sendo capaz de identificar de maneira correta aproximadamente $88,8 \%$ dos casos. 
Já em relação ao processo de identificação do Qualis para Trabalho Completo em Conferência (TCC), o resultado foi inferior aos obtidos no processo de identificação para AP. Isto ocorre devido aos TCC não possuírem ISBN, obrigando que a busca pelo Qualis seja realizada através do nome do local de publicação. O que dificulta a identificação, devido a falta de padronização no preenchimento deste campo no Currículo Lattes.

Após analisar as 4092 publicações referentes ao elemento TCC pertencente aos 47 pesquisadores. Conclui-se que 47,3\% tiveram seus Qualis classificados de maneira correta. Outras 823 publicações, ou seja, 20,1\% não tiveram seus Qualis identificados devido a não terem seus veículos de divulgação presentes na lista da Capes, e por isso sendo considerados corretamente avaliados, já que de fato não possuem Qualis.

Outros 3,9\% aparentam terem sido preenchidos de maneira incorreta, pois continham palavras ou frases que não representavam nenhum tipo de sigla ou nome de conferências. E outras 19 publicações, não tiveram seus locais de divulgação preenchidos.

Desta forma pode-se concluir que $71,8 \%$ das publicações realizadas em conferências, foram de fato identificadas corretamente. O restante não pode ser classificado devido a dois motivos principais, o primeiro representa um total de $12,4 \%$ e está relacionado a erros de escrita, já que diversos veículos de divulgação tiveram seus nomes preenchido com erros, dificultando assim a comparação.

O segundo motivo foi devido a falta de padronização no preenchimentos dos locais de publicação, representando um total de 15,8\%. Devido ao preenchimento deste campo no Currículo Lattes ser de maneira livre, existe muita divergência no preenchimento das informações, como por exemplo, termos acrescentados ou removidos e palavras fora de ordem.

Estes problemas dificultam bastante a identificação do Qualis das publicações referentes ao elemento TCC. O gráfico mostrado na Figura 3 representam o resultado do processo de identificação do Qualis para as publicações do elemento TCC.

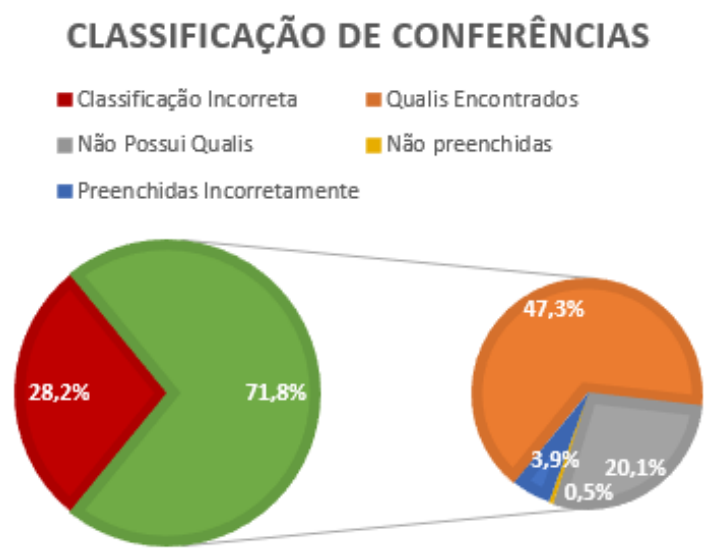

Figura 3. Classificação do Qualis para Trabalhos Completos em Conferências.

Observando o gráfico da Figura 3, pode-se considerar que o processo de identificação do Qualis para Trabalhos Completos em Conferências, é satisfatório, alcançando um total de $71,8 \%$ de publicações identificadas corretamente, embora acredita-se que seja possível melhorar estes resultados futuramente. 
Também foi realizado outro experimento com o intuito de analisar o desempenho do Rep-Index e da Pontuação Qualis. Para isso utilizou-se todos os 469 docentes presentes na base de dados. Durante o experimento os currículos destes docentes foram colocados em 15 grupos distintos. Onde 12 deles representavam as universidades com as quais os docentes estavam vinculados. E outros 3 grupos representando o conceito Capes a qual estes programas fazem parte.

O objetivo da criação destes grupos foi avaliá-los, de forma a verificar se a Pontuação Qualis e o Rep-Index, estão gerando valores condizentes com o esperado para cada grupo. Sendo que quanto maior o conceito Capes de um programa, maior deverá ser o desempenho médio de seus docentes segundo a Capes [CAPES 2016a].

De forma a analisar a correlação entre os índices Pontuação Qualis e Rep-Index, com o conceito Capes de cada programa. Foi necessário obter a média dos valores para cada um dos três grupos que representam tais conceitos. Dessa forma pode-se verificar que de fato existe uma correlação entre a nota recebida e o conceito capes da qual o docente faz parte, como pode ser observado nas Figruas 4 e 5.

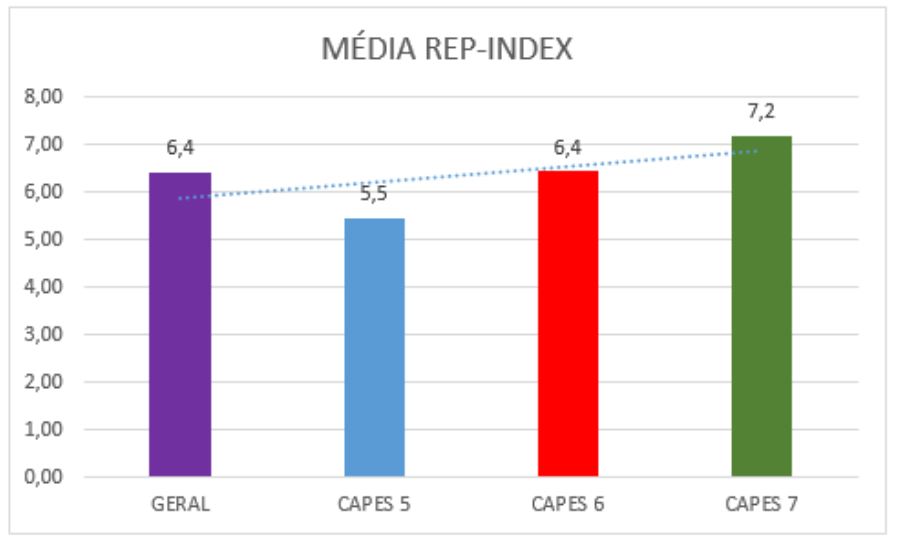

Figura 4. Média do Rep-Index por conceito Capes.

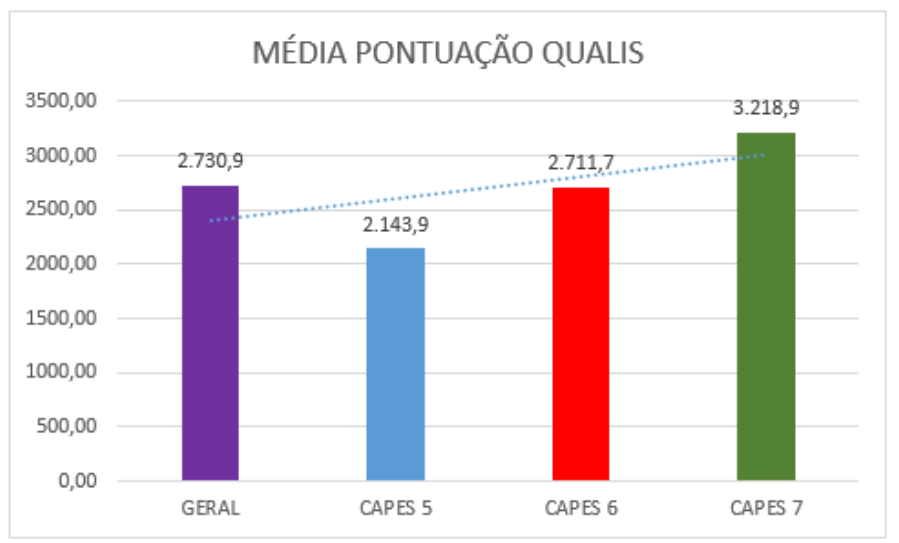

Figura 5. Média da Pontuação Qualis por conceito Capes.

Analisando os gráficos das Figuras 4 e 5, fica claro que quanto maior o conceito do programa, maior a média da Pontuação Qualis e do Rep-index de seus docentes. Porém 
embora a produtividade dos docentes estejam diretamente ligado ao conceito de seus programas, este não é o único fator determinante. Como pode ser observado analisando as notas de cada um dos 12 programas, como é mostrado na Tabela 3.

Tabela 3. Descrição dos grupos que representam programas de pós-graduação.

\begin{tabular}{|c|c|c|c|c|}
\hline Conceito & Sigla & Rep-Index & Pontuação Qualis & Qtd. de Docentes \\
\hline 5 & A5 & 6,20 & 2853,75 & 24 \\
\hline 5 & B5 & 4,55 & 2020,95 & 21 \\
\hline 5 & C5 & 4,73 & 1613,91 & 23 \\
\hline 5 & D5 & 5,82 & 2109,67 & 45 \\
\hline 5 & E5 & 5,50 & 2112,17 & 23 \\
\hline 6 & F6 & 6,18 & 2783,23 & 65 \\
\hline 6 & G6 & 5,79 & 2098,97 & 34 \\
\hline 7 & H6 & 7,07 & 2964,45 & 64 \\
\hline 7 & I7 & 8,42 & 3465,83 & 24 \\
\hline 7 & J7 & 7,09 & 3399,89 & 45 \\
\hline 7 & K7 & 7,94 & 3598,21 & 53 \\
\hline 7 & L7 & 5,83 & 2506,98 & 48 \\
\hline
\end{tabular}

Analisando os valores da Tabela 3, é possível notar que existe programas, cuja suas notas ficaram consideravelmente abaixo ou acima da média de seus conceitos. Mostrando que embora a produtividade dos docentes de um programa de pós-graduação seja um importante quesito para a avaliação da Capes, outros critérios de avaliação também exercem grande influência na decisão.

Com isso pode-se observar também, que entre as 12 universidades, apenas 4 delas obtiveram uma pontuação para o Rep-Index acima da média, que foi de 6,43, são elas: H6, I7, J7, K7. Já a universidade L7 embora esteja conceituada no nível mais alto de avaliação da Capes, obteve uma nota de 5,83, ficando assim abaixo da média geral.

\section{Conclusão}

A proposta de desenvolver uma ferramenta capaz de mapear e avaliar os currículos cadastrados na Plataforma Lattes, de forma transparente, rápida e ampla, idependente da área de conhecimento, foi realizada com sucesso, com a criação do SWAP-BR. Neste trabalho foram apresentados suas funcionalidades, caracteristica e resultados obtidos através de sua utilização.

Através dos testes e experimentos foi possível realizar uma análise completa dos docentes de cada um dos programas de pós-graduação. E verificar que a qualidade do trabalho de um pesquisador é fundamental para a definição dos conceitos dos programas, junto a Capes.

Como trabalhos futuros pretende-se automatizar o processo de download e upload do Currículo Lattes para o SWAP-BR, agilizando ainda mais o processo de avaliação. Utilizar algoritmos de inteligência artificial, para definir os elementos do Rep-Model que serão avaliados em cada área e também a fim de definir os pesos de cada um dos elementos de forma automática. 


\section{Referências}

CAPES, C. d. A. d. P. d. N. S. (2013a). Documento de Área 2013 - ciência da computação.

CAPES, C. d. A. d. P. d. N. S. (2013b). Regulamento para a avaliação trienal 2013 (20102012).

CAPES, C. d. A. d. P. d. N. S. (2016a). Documento de Área 2016 - ciência da computação.

CAPES, C. d. A. d. P. d. N. S. (2016b). Plataforma sucupira.

Cervi, C. R. (2013). Rep-index : uma abordagem abrangente e adaptável para identificar reputação acadêmica. UFRGS Lume Repositorio Digital.

Cervi, C. R., Galante, R., and de Oliveira, J. P. M. (2011). Identificando a reputação de pesquisadores usando um modelo de perfil adaptativo. In CONGRESSO DA SOCIEDADE BRASILEIRA DE COMPUTACAO, volume 31.

Cervi, C. R., Galante, R., and De Oliveira, J. P. M. (2013a). Comparing the reputation of researchers using a profile model and scientific metrics. In Computational Science and Engineering (CSE), 2013 IEEE 16th International Conference on, pages 353-359. IEEE.

Cervi, C. R., Galante, R., and Oliveira, J. (2012). An adaptive approach for identifying reputation of researchers. In IADIS International Conference on WWW/Internet, Marid.

Cervi, C. R., Galante, R., and Oliveira, J. d. (2013b). Application of scientific metrics to evaluate academic reputation in different research areas. World Academy of Science, Engineering and Technology, International Journal of Social, Behavioral, Educational, Economic, Business and Industrial Engineering, 7(10):2778-2788.

CNPq, C. N. d. D. C. e. T. (2016). Sobre a plataforma lattes.

Egghe, L. (2006). An improvement of the h-index: The g-index. ISSI newsletter, 2(1):89.

Garfield, E. (2010). The evolution of the science citation index. International microbiology, 10(1):65-69.

Google (2004). Google acadêmico.

Hirsch, J. E. (2005). An index to quantify an individual's scientific research output. Proceedings of the National academy of Sciences of the United States of America, pages 16569-16572.

Khaliluzzaman, M. and Chowdhury, I. I. (2016). Pre and post controller based mvc architecture for web application. In 2016 th International Conference on Informatics, Electronics and Vision (ICIEV), pages 552-557.

Mena-Chalco, J. P., Junior, C., and Marcondes, R. (2009). Scriptlattes: an open-source knowledge extraction system from the lattes platform. Journal of the Brazilian Computer Society, 15(4):31-39.

Microsoft (2012). Microsoft academic.

Page, L., Brin, S., Motwani, R., and Winograd, T. (1999). The pagerank citation ranking: Bringing order to the web. Technical report, Stanford InfoLab. 